\title{
Evidence of two-center interference in high-order harmonic generation from $\mathrm{CO}_{2}$
}

\author{
Anh-Thu Le, ${ }^{1, *}$ X.-M. Tong, ${ }^{2}$ and C. D. Lin ${ }^{1}$ \\ ${ }^{1}$ Department of Physics, Cardwell Hall, Kansas State University, Manhattan, Kansas 66506, USA \\ ${ }^{2}$ Institute of Materials Science, Graduate School of Pure and Applied Science and Center for Computational Sciences, \\ University of Tsukuba, 1-1-1 Tennodai, Tsukuba, Ibaraki 305-8573, Japan
}

(Received 9 February 2006; published 6 April 2006)

\begin{abstract}
Two recent pump-probe experiments on the high-order harmonic generation (HHG) from partially aligned $\mathrm{CO}_{2}$ molecules have shown that the HHG yields versus the ionization rates as functions of the pump-probe delay time exhibit inverted modulation. The inversion has been attributed to the quantum interference in the recombination process from the two oxygen centers. Using the Lewenstein model to calculate HHG from fixed $\mathrm{CO}_{2}$ molecules and taking into account the partial alignment of the molecules versus the time delay, we interpret that the inversion is due to the degree of overlap between the partially aligned molecules and the angular dependence of the HHG yield. We also note that the harmonic order where inversion occurs may change with probe laser intensity due to the depletion of the ground state.
\end{abstract}

DOI: 10.1103/PhysRevA.73.041402

High-order harmonic generation (HHG) is one of the most studied phenomena in intense laser-matter interaction. HHG from atoms is well understood as a three-step process [1-3]. Within this model, the active electron first tunnels through the potential barrier formed by the atomic Coulomb potential and the laser field. After the laser field reverses its direction the released electron has a probability of returning back to the parent ion where it may recombine to emit a high-energy photon.

For the HHG from a molecule, an interesting interference effect has been predicted first from the calculated HHG spectra of a model two-dimensional (2D) $\mathrm{H}_{2}{ }^{+}$by solving numerically the time-dependent Schrödinger equation (TDSE), assuming that the nuclei are fixed in space, by Lein et al. [4]. They found pronounced minima in the HHG power spectra which they later interpreted as the interference of the emissions from the two atomic centers, when the wavelength of the returning electron matches the typical distance between the nuclei. Similar interference has been found later in the realistic three-dimensional (3D) model of $\mathrm{H}_{2}^{+}$by Lein et al. [5] and Kamta and Bandrauk [6] under the same fixed-nuclei approximation. Telnov and Chu [7] have also calculated the HHG for $\mathrm{H}_{2}{ }^{+}$aligned along the laser polarization direction, but no signature of interference was reported.

On the experimental side, it has been of great interest to study the HHG yield for molecules that are at least partially aligned. This is achieved by exposing molecules to a short, relatively weak pulse to create a rotational wave packet. This wave packet rephases after the pulse is over and the molecules are strongly aligned periodically at intervals separated by their fundamental rotational period [8]. To observe the alignment dependence of HHG, a second short laser pulse is then used to generate HHG at different short intervals when the molecules undergo rapid change in their alignment.

In a recent paper, Kanai et al. [9] performed such pumpprobe experiments on $\mathrm{N}_{2}, \mathrm{O}_{2}$, and $\mathrm{CO}_{2}$ using an 800-nm laser. By comparing the 23rd harmonic signals versus the

*Email address: atle@phys.ksu.edu
PACS number(s): $33.80 . R v, 42.65 . K y$

ionization yields as functions of the time delay between the two laser pulses (same polarization direction), they found that the two curves follow each other for $\mathrm{N}_{2}$ and $\mathrm{O}_{2}$, i.e., when the ionization yield is maximum (minimum) the $\mathrm{HHG}$ yield is also maximum (minimum). Noting that for $\mathrm{N}_{2}$ the ionization is maximal along the molecular axis [10], these results are in fact in good agreement with the measurements by Itatani et al. [11]. These results imply that the HHG yield and the ionization rate for each molecule follow the same alignment dependence and the recombination step in the HHG mechanism does not introduce additional alignment dependence. In contrast, they observed inverted modulation in the HHG signal for the 23rd harmonic from $\mathrm{CO}_{2}$ as a function of pump-probe delay time. That is, they found minima in HHG emission at maximum ionizations and vice versa. Most recently, Vozzi et al. [12] also confirmed the inverted modulation from $\mathrm{CO}_{2}$, but for the 33rd harmonic. Both groups attributed the origin of the inversion to the interference of the photon emission from the two oxygen centers in $\mathrm{CO}_{2}$ which are separated by about twice the distance between the two nuclei in $\mathrm{O}_{2}$ where no inversion was found.

Can one indeed conclude that the inverted modulation in the $\mathrm{HHG}$ versus the ionization spectra in $\mathrm{CO}_{2}$ is a manifestation of the interference in the recombination process? Note that the harmonic order for the interference minimum depends on the angle between the molecular axis and the laser polarization direction. In both experiments the molecules are only partially aligned by the pump pulse (thus the measured HHG signals should be averaged over the angular distributions of the molecules), and it is not clear that the minimum predicted from the interference model in fixed nuclei approximation can survive after the average. Furthermore, if the interference model is indeed the correct interpretation, then it should occur at the same harmonic order, independent of the laser intensity of the probe pulse. In the experiments by Kanai et al. and Vozzi et al., the inversion was found at different harmonic orders.

In this paper, we show that there are other possible interpretations for the observed inverted modulation in $\mathrm{CO}_{2}$. In order to compare with experiments, in our analysis the anisotropic angular distributions of the molecules at each time 


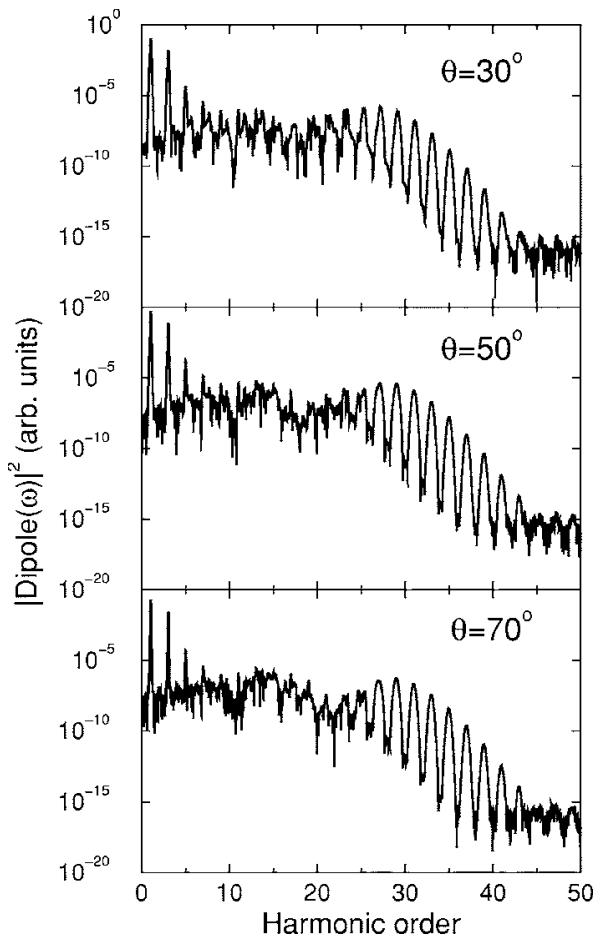

FIG. 1. HHG power spectra for selective fixed alignment angles $\theta=30^{\circ}$ (top), $50^{\circ}$ (middle), and $70^{\circ}$ (bottom) at laser intensity of $1.5 \times 10^{14} \mathrm{~W} / \mathrm{cm}^{2}$. For other laser parameters, see text.

delay after the pump pulse are taken into account in obtaining the calculated HHG spectra and the ionization yields. To calculate the HHG spectra for a fixed alignment, we used the Lewenstein model [3] for molecules as extended in Zhou et al. [13]. This model has been shown [13] to be able to interpret the experimental results reported so far for $\mathrm{N}_{2}$ and $\mathrm{O}_{2}$ $[9,11,14]$.

The Lewenstein model for a molecule aligned along the $z$ axis in a laser field $E(t)$, linearly polarized on the $y-z$ plane with an angle $\theta$ with respect to the molecular axis can be written in the form (atomic units $m=e=\hbar=1$ are used throughout the paper, unless otherwise indicated)

$$
\begin{aligned}
r(t)= & i \int_{0}^{\infty} d \tau\left(\frac{\pi}{\epsilon+i \tau / 2}\right)^{3 / 2}\left[\sin \theta d_{y}^{*}(t)+\cos \theta d_{z}^{*}(t)\right] \\
& \times\left[\sin \theta d_{y}(t-\tau)+\cos \theta d_{z}(t-\tau)\right] E(t-\tau) \\
& \times \exp \left[-i S_{s t}(t, \tau)\right] a^{*}(t) a(t-\tau)+\text { c.c. },
\end{aligned}
$$

where $\quad \boldsymbol{d}(t) \equiv \boldsymbol{d}\left[\left(\boldsymbol{p}_{s t}(t, \tau)-\boldsymbol{A}(t)\right], \quad \boldsymbol{d}(t-\tau) \equiv \boldsymbol{d}\left[\left(\boldsymbol{p}_{s t}(t, \tau)\right.\right.\right.$ $-\boldsymbol{A}(t-\tau)]$ are the transition dipole moments between the ground state and the continuum state, and $\boldsymbol{p}_{s t}(t, \tau)$ $=\int_{t-\tau}^{t} \boldsymbol{A}\left(t^{\prime}\right) d t^{\prime} / \tau$ is the canonical momentum at the stationary points, with $\boldsymbol{A}$ the vector potential. The quasiclassical action at the stationary points for the electron propagating in the laser field is

$$
S_{s t}(t, \tau)=\int_{t-\tau}^{t}\left(\frac{\left[\mathbf{p}_{s t}(t, \tau)-\mathbf{A}\left(t^{\prime}\right)\right]^{2}}{2}+I_{p}\right) d t^{\prime}
$$

where $I_{p}$ is the ionization potential of the molecule. In Eq. (1), $a(t)$ is introduced to account for the ground-state depletion.
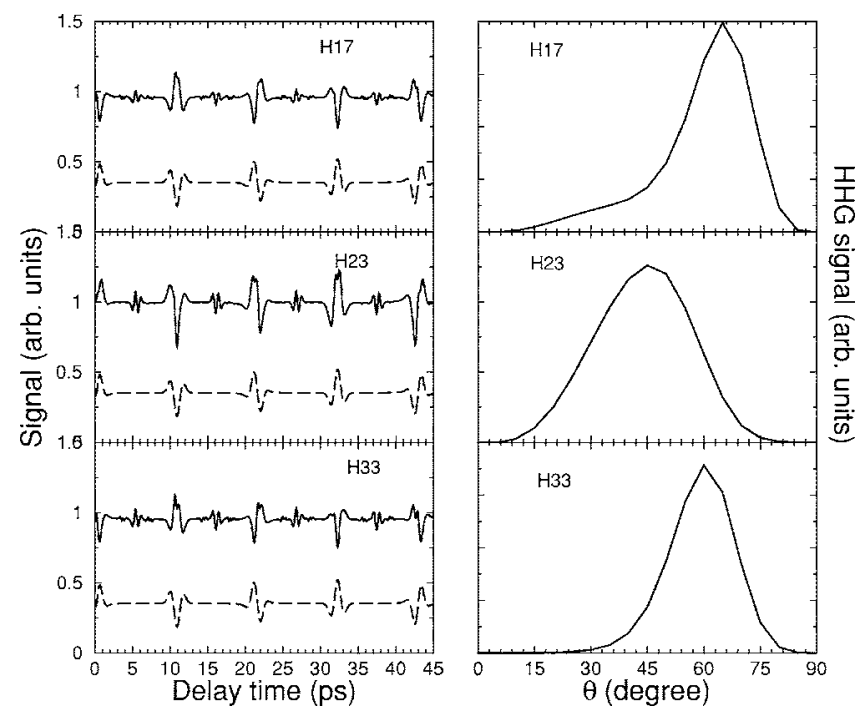

FIG. 2. Time evolution of the selective harmonics (left column) and their alignment dependence (right column) at the probe laser intensity of $2 \times 10^{14} \mathrm{~W} / \mathrm{cm}^{2}$. For other laser parameters, see text. Dashed curves correspond to the evolution of $\left\langle\cos ^{2} \theta\right\rangle$.

In Fig. 1, we plot the $\mathrm{HHG}$ power spectra calculated within our extended Lewenstein model from $\mathrm{CO}_{2}$ molecules aligned at three fixed angles $\theta=30^{\circ}, 50^{\circ}$, and $70^{\circ}$. The ground-state electronic wave function of $\mathrm{CO}_{2}$ was obtained from the GAMESS code [15]. In order to account for the depletion of the ground state, we approximate the groundstate amplitude by $a(t)=\exp \left[-\int_{-\infty}^{t} W\left(t^{\prime}\right) / 2 d t^{\prime}\right]$, with the ionization rate $W\left(t^{\prime}\right)$ obtained from the molecular tunneling ionization (MO-ADK) theory [16]. For this calculation, we took a Gaussian pulse of 30 fs duration [full width at half maximum (FWHM)], peak intensity of $1.5 \times 10^{14} \mathrm{~W} / \mathrm{cm}^{2}$, and a mean wavelength of $800 \mathrm{~nm}$. We note from these figures that there is no obvious "interference minimum" for the harmonics above about $N=25$ for all the 31 alignment angles between $0^{\circ}$ and $90^{\circ}$ that we have calculated.

Based on these results does it mean that one should not expect inverted modulation in the pump-probe experiments? Figure 2 shows the HHG signal as a function of delay time for the 17th (left, top panel), 23rd (left, middle panel), and 33rd harmonics (left, bottom) at the probe laser intensity of $2 \times 10^{14} \mathrm{~W} / \mathrm{cm}^{2}$. The signals have been normalized to that of the isotropic molecular distribution. For reference, we also plot here the evolution of $\left\langle\cos ^{2} \theta\right\rangle$ (dashed curves), as it is a measure of the degree of alignment. We note that the ionization signals follow closely the evolution of $\left\langle\cos ^{2} \theta\right\rangle$ (see Fig. 3 below). For calculating the time evolution of the alignment distribution of the molecules we use the rotor model for diatomic molecules $[17,18]$

$i \frac{\partial \Psi(\theta, \phi, t)}{\partial t}=\left[B \boldsymbol{J}^{2}-\frac{E(t)^{2}}{2}\left(\alpha_{\|} \cos ^{2} \theta+\alpha_{\perp} \sin ^{2} \theta\right)\right] \Psi(\theta, \phi, t)$,

where $B$ is the rotational constant, $\alpha_{\|}$and $\alpha_{\perp}$ are the anisotropic polarizabilities in parallel and perpendicular directions 


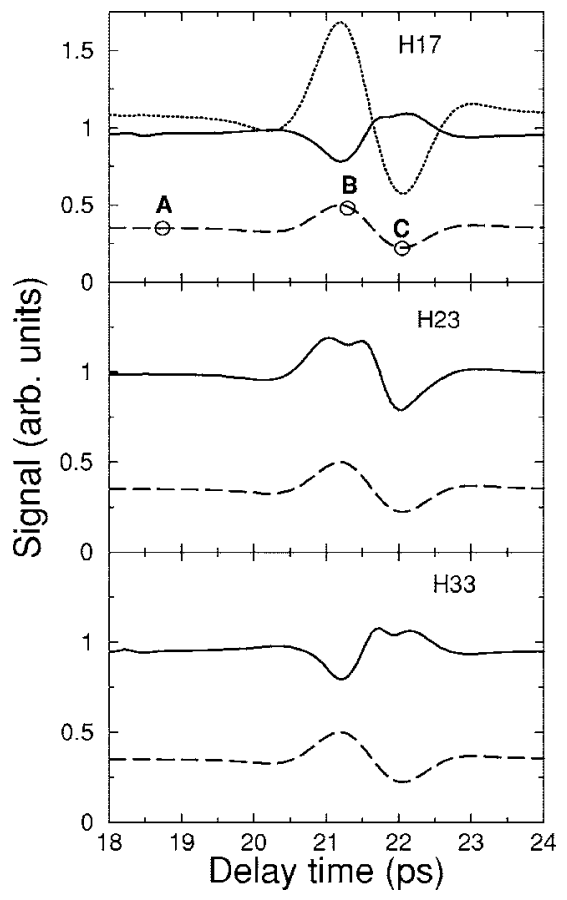

FIG. 3. Same as Fig. 2 but near half revival. On the top panel the ionization signal (dotted curve) is also shown.

with respect to the molecular axis, respectively. The TDSE (3) with $B=0.39 \mathrm{~cm}^{-1}, \alpha_{\|}=4.05 \AA^{3}$, and $\alpha_{\perp}=1.95 \AA^{3}[19]$ is then solved for each initial rotational state $|J M\rangle$ using the split-operator method [20]. The pump laser $E(t)$ for aligning the molecules is assumed to be a Gaussian pulse, with a duration of $50 \mathrm{fs}$ and peak intensity of $3 \times 10^{13} \mathrm{~W} / \mathrm{cm}^{2}$. The pump and probe pulses are polarized parallel. We also assume the Boltzmann distribution of the rotational levels at the initial time. The rotational temperature of $\mathrm{CO}_{2}$ molecules is taken to be $40 \mathrm{~K}$. One can see clearly the inverted modulation for the 17th and 33rd harmonics. This is in qualitative agreement with the results by Kanai et al. [9]. The right panels show the alignment dependence of these harmonics. We notice that for the 17th and 33rd harmonics, the distribution is peaked at the angles greater than about $50^{\circ}$, whereas it is peaked at about $45^{\circ}$ for the 23 rd harmonic. We will come back to this point later. Note that the calculations by de Nalda et al. [21] indicate that the alignment dependence of HHG from $\mathrm{CO}_{2}$ for lower orders $(N=9-17)$ have peaks at angles greater than $45^{\circ}$ too.

Let us try to understand these results. In Fig. 3 we show the closeup of the time evolution for the HHG near half revival $(t=21.3 \mathrm{ps})$. We also plot in the upper panel the ionization yield (dotted curve), normalized to that of the isotropic distribution. Recall that the time evolution of the HHG yield for a particular harmonic can be written in the form

$$
g_{2 n+1}(t)=2 \pi \int_{0}^{\pi} \rho(\theta, t) \bar{g}_{2 n+1}(\theta) d \theta,
$$

where $\rho(\theta, t)$ is the weighted angular distribution (i.e., multiplied by $\sin \theta)$ of the molecules, $\bar{g}_{2 n+1}(\theta)$ is the HHG yield for the $(2 n+1)$-th harmonic from the molecules aligned at a
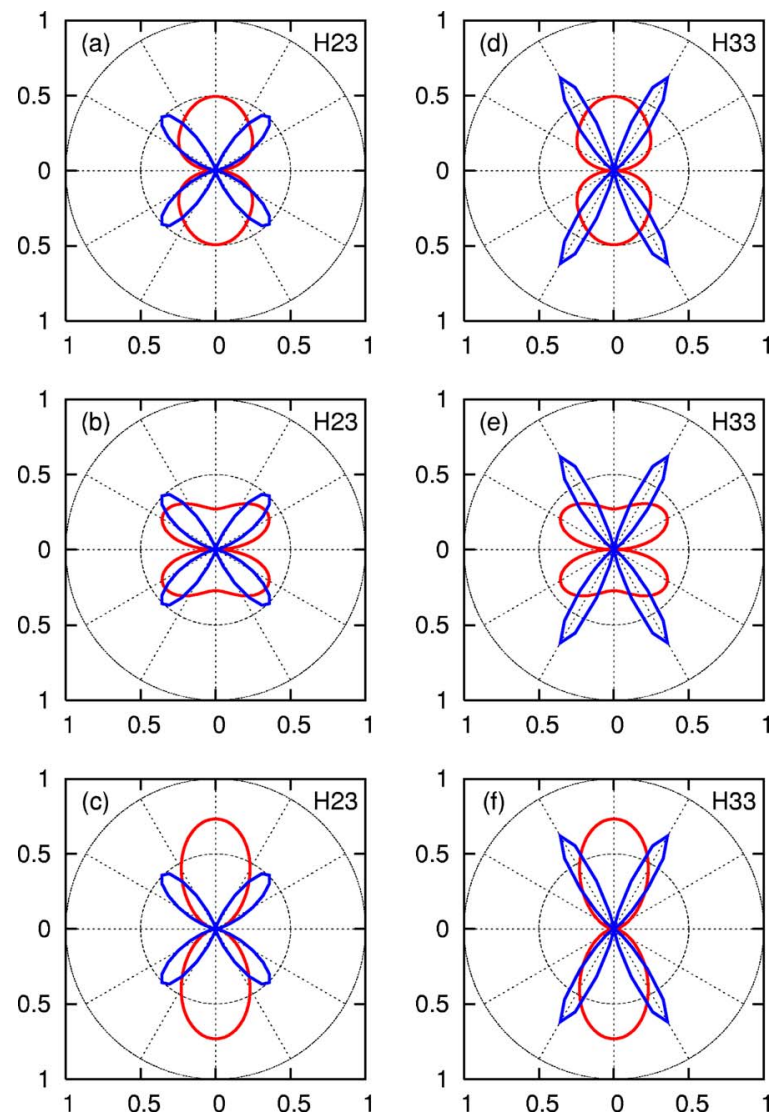

FIG. 4. (Color online) Polar plot of the weighted alignment distribution of the molecules [red (light) curves] at different time delays $t=18.7$ (top), 21.3 (middle), and $22.1 \mathrm{ps}$ (bottom) (see also Fig. 3). Also plotted is the alignment dependence [blue (dark) curves] for the 23rd harmonic (left column) and the 33rd harmonic (right column). The laser parameters are the same as for Fig. 2. Laser polarization is along the horizontal axes.

fixed angle $\theta$, and $t$ is the delay time. Here we already account for the fact that the system has azimuthal symmetry. Figure 4 shows the polar plot of the weighted angular distribution [red (light) curves] at three different pump-probe delays: 18.7 (upper panels), 21.3 (middle panels), and $22.1 \mathrm{ps}$ (bottom panels), denoted, respectively, as A, B, and C in Fig. 3. Also plotted are the HHG yields [blue (dark) curves] for the 23rd harmonic (left column) and the 33rd harmonic (right column). Let us now consider the 23rd harmonic. Due to the symmetry, we need only consider $\theta$ in the range $\left[0^{\circ}-90^{\circ}\right]$. First, we notice that the HHG yield is well localized in the angular range $\left[30^{\circ}-60^{\circ}\right]$, as can also be seen from Fig. 2 (right, middle panel). For that range of alignment, as can be seen from the figure, the weighted alignment distribution is largest for B and smallest for $\mathrm{C}$. Therefore, according to Eq. (4), the time evolution of HHG is not inverted, which is consistent with the results in Fig. 2. In contrast, the 33rd harmonic yield is even narrower with the peak near $60^{\circ}$. For this small range of alignment, the weighted alignment distribution is smallest for B and largest for C, which leads to the inverted modulation, according to Eq. (4).

At lower probe laser intensities, the depletion effect becomes weaker. As a consequence, the HHG peaks are 

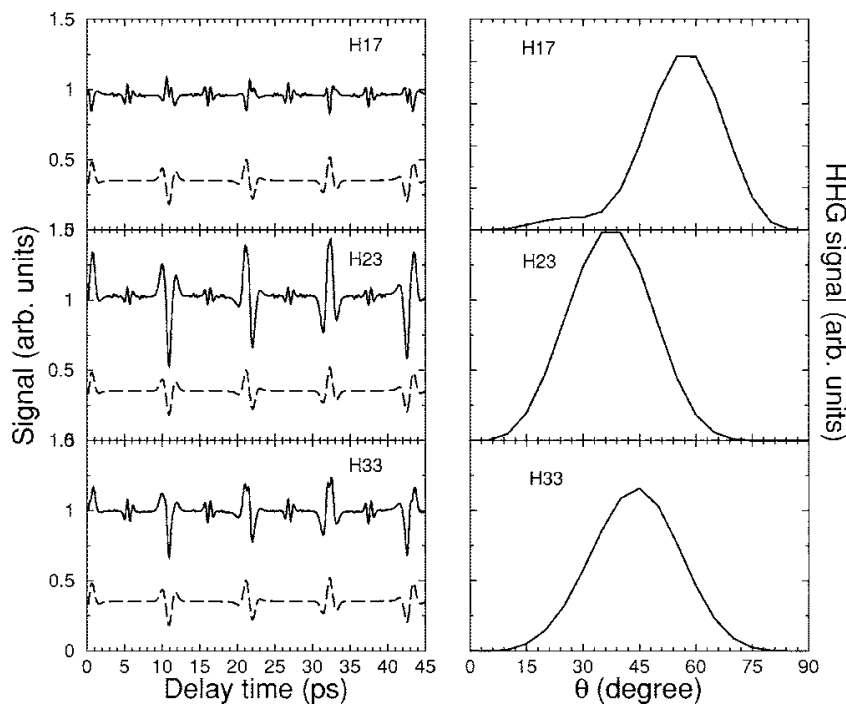

FIG. 5. Same as Fig. 2 but for the probe laser intensity of $1 \times 10^{14} \mathrm{~W} / \mathrm{cm}^{2}$.

shifted to the smaller alignment angles. According to the discussion above, this would lead to a drastic change in the behavior for the time evolution of HHG yield. In fact, at $1.5 \times 10^{14} \mathrm{~W} / \mathrm{cm}^{2}$, the results still do not differ qualitatively from that of $2 \times 10^{14} \mathrm{~W} / \mathrm{cm}^{2}$. For intensity of $1 \times 10^{14} \mathrm{~W} / \mathrm{cm}^{2}$, we plot in Fig. 5 the HHG yields as functions of delay time for three harmonics of the 17th, 23rd, and 33rd orders (left panels). Other laser parameters are the same as for Fig. 2. As one can see, the HHG dependence on the alignment (right panels) is shifted towards smaller angles, as compared to that from Fig. 2. As a consequence, the 33rd harmonic is not inverted. Thus one may suspect that the different harmonics where the inversion occurs in the experiment of Kanai et al. [9] and of Vozzi et al. [12] are due to the different probe intensities used. Future experiments with several probe laser intensities are therefore highly desirable in order to distinguish the present interpretation from the interference model.

In conclusion, we have shown that inverted modulations are not necessarily associated with the interference picture which has been used to explain the results of the recent experiments by Kanai et al. [9] and Vozzi et al. [12]. We believe that the main conclusion is not limited to the strongfield approximation that we used. The angular distributions of the partially aligned molecules by the pump pulse should be taken into account in the interpretation of the experimental HHG data in pump-probe experiments. Our results also show that the angular dependence and the time evolution of the HHG yield are influenced strongly by the depletion of the ground state and are, therefore, sensitive to the probe laser intensity. Finally, we emphasize that the present calculations are limited to the Lewenstein model. Full $a b$ initio calculations of HHG for such molecules are not readily available anytime soon. Thus further experiments are needed to shed more light on the possible limitation of the present model in predicting the alignment dependence of HHG from molecules.

A.T. would like to thank Dr. X. X. Zhou and Dr. Z. X. Zhao for the discussions on the HHG from $\mathrm{N}_{2}$ and $\mathrm{O}_{2}$. This work was supported by the Chemical Sciences, Geosciences and Biosciences Division, Office of Basic Energy Sciences, Office of Science, U. S. Department of Energy.
[1] K. C. Kulander, K. J. Schafer, and J. L. Krause, in Super Intense Laser-Atom Physics, edited by B. Piraux, NATO Advanced Studies Institute (Plenum, New York, 1993), Vol. 316, p. 95.

[2] P. B. Corkum, Phys. Rev. Lett. 71, 1994 (1993).

[3] M. Lewenstein, Ph. Balcou, M. Yu. Ivanov, A. L'Huillier, and P. B. Corkum, Phys. Rev. A 49, 2117 (1994).

[4] M. Lein, N. Hay, R. Velotta, J. P. Marangos, and P. L. Knight, Phys. Rev. Lett. 88, 183903 (2002).

[5] M. Lein, P. P. Corso, J. P. Marangos, and P. L. Knight, Phys. Rev. A 67, 023819 (2004).

[6] G. L. Kamta and A. D. Bandrauk, Phys. Rev. A 71, 053407 (2005).

[7] D. A. Telnov and S.-I. Chu, Phys. Rev. A 71, 013408 (2005).

[8] H. Stapelfeldt and T. Seideman, Rev. Mod. Phys. 75, 543 (2003).

[9] T. Kanai, S. Minemoto, and H. Sakai, Nature (London) 435 , 470 (2005).

[10] I. V. Litvinyuk, K. F. Lee, P. W. Dooley, D. M. Rayner, D. M. Villeneuve, and P. B. Corkum, Phys. Rev. Lett. 90, 233003 (2003).

[11] J. Itatani, D. Zeidler, J. Levesque, M. Spanner, D. M. Ville- neuve, and P. B. Corkum, Phys. Rev. Lett. 94, 123902 (2005).

[12] C. Vozzi, F. Calegari, E. Benedetti, J.-P. Caumes, G. Sansone, S. Stagira, M. Nisoli, R. Torres, E. Heesel, N. Kajumba, J. P. Marangos, C. Altucci, and R. Velotta, Phys. Rev. Lett. 95, 153902 (2005).

[13] X. X. Zhou, X. M. Tong, Z. X. Zhao, and C. D. Lin, Phys. Rev. A 71, 061801(R) (2005); 72, 033412 (2005).

[14] J. Itatani, J. Levesque, D. Zeidler, H. Niikura, H. Pepen, J. C. Kieffer, P. B. Corkum, and D. M. Villeneuve, Nature (London) 432, 867 (2004).

[15] M. W. Schmidt et al., J. Comput. Chem. 14, 1347 (1993).

[16] X. M. Tong, Z. X. Zhao, and C. D. Lin, Phys. Rev. A 66, 033402 (2002).

[17] T. Seideman, J. Chem. Phys. 103, 7887 (1995).

[18] J. Ortigoso, M. Rodriguez, M. Gupta, and B. Friedrich, J. Chem. Phys. 110, 3870 (1999).

[19] J. O. Hirschfelder, C. F. Curtiss, and R. B. Bird, Molecular Theory of Gases and Liquids (Wiley, New York, 1954).

[20] X. M. Tong and S. I. Chu, Chem. Phys. 217, 119 (1997).

[21] R. de Nalda, E. Heesel, M. Lein, N. Hay, R. Velotta, E. Springate, M. Castillejo, and J. P. Marangos, Phys. Rev. A 69, 031804(R) (2004). 